\title{
Commentary: Protective Effects of Isorhamnetin on N2a Cell Against Endoplasmic Reticulum Stress-induced Injury Is Mediated by PKCe
}

Lingyu Qiu, Huiqiang Lu*

Jiangxi Province Key Laboratory of Developmental Biology of Organs, Jiangxi Engineering laboratory of Zebrafish Modeling and Drug Screening for Human Diseases, Center for Developmental biology of Jinggangshan University, College of life sciences, Jinggangshan University, Ji'an, Jiangxi 343009, China

Article Info

\section{Article Notes}

Received: September 15, 2017

Accepted: October 13, 2017

\section{${ }^{*}$ Correspondence:}

Dr. Huiqiang Lu, College of life sciences; Jinggangshan University, No. 28 Xueyuan Road, Qingyuan District, Ji'an, Jiangxi 343009, China, Tel: (+86) 7968116 182; Fax: (+86) 7968116 182, Email: luhq2@126.com

C) $2017 \mathrm{Lu} \mathrm{H}$. This article is distributed under the terms of the Creative Commons Attribution 4.0 International License

\section{ABSTRACT}

Neuronal apoptosis is an important pathophysiological factor of Alzheimer's disease (AD). Inhibition of endoplasmic reticulum stress (ERS)-induced neuronal apoptosis is an effective strategy to deal with AD. In this commentary, we summarize the relationship between AD and ERS injury-induced neuronal apoptosis, and highlight the protective effects and mechanism of isorhamnetin (Iso) against ERS-induced injury in N2a cells. Moreover, this commentary discusses the recent findings in the role of Iso in other diseases.

Alzheimer's disease (AD) is a chronic neurodegenerative disease, and the fourth leading cause of death, behind heart disease, cancer and stroke ${ }^{1}$. The clinical manifestations of AD include deteriorating cognitive function and memory, a decline in functioning in daily life activities, anda variety of neuropsychiatric symptoms and behavioral disorders ${ }^{2,3}$. The risk of $\mathrm{AD}$ gradually increases with age. There are more than 30 million $\mathrm{AD}$ patient living in the world today ${ }^{4,5}$.

The etiology and pathogenesis of $A D$ is not clear. AD is characterized by the accumulation of misfolded $\beta$-amyloid proteins caused by various genetic and environmental factors. These $A \beta$-plaques are associated with several pathological changes including the aggregation of misfolded proteins, formation of tissue precipitation, intracellular calcium disturbance, DNA fragmentation and lipid peroxidation. Together, these factors induce neuronal apoptosis and necrosis ${ }^{6}$. Thus, inhibiting neuron apoptosis is an effective way to prevent AD.

The endoplasmic reticulum (ER) is theprincipal organelle for protein synthesis, protein folding and maintenance of calcium homeostasis ${ }^{7}$. Numerous stimuli, such as hypoxia and oxidative stress, can perturb calcium balance, and promote accumulation of unfolded and misfolded proteins in the ER lumen. This is known as endoplasmic reticulum stress $(E R S)^{8}$. ERS is one of the key drivers of apoptosis. ERS induces apoptosis, through activation of the ASK and p38 MAPK signal pathway, trigger the kinase function of IRE1 $1^{9-11}$. Additionally, p38MAPK is reported to promote phosphorylation and activation of pro-apoptotic protein $\mathrm{Bax}^{12,13}$.

Recent studies suggest that AD pathology is associated with ERS ${ }^{14}$. ERS generates a toxic environment in neurons, which in turn activates endoplasmic reticulum pathways that ultimately cause apoptosis ${ }^{15}$. Thus, inhibition of ERS injury may be an effective strategy to prevent AD. 
Our study "Protective Effects of Isorhamnetin on N2a Cell Against Endoplasmic Reticulum Stress-induced Injury Is Mediated by PKC $\varepsilon$ " suggests that Isorhamnetin (Iso) serves as a neuroprotective against ERS-induced apoptosis.

Iso has been reported to inhibit anoxia/reoxygenationinduced apoptosis by STAT1. In our study, we identify the protective effect and molecular mechanism of Iso inhibition of ERS-induced apoptosis in N2a cells. During ERS, GRP78 is released in the lumen and IRE $1 \alpha$ and ATF 6 are activated, resulting in up-regulation of ERS marker geneGRP78.Thus, we used GRP78protein levels to estimate ERS injury and determine optimal concentration and pretreatment time for Iso.

ERS also results in $\mathrm{Ca}^{2+}$ release from the $\mathrm{ER}$ into the cytosol, which induces ROS burst, and eventually neuronal apoptosis $^{16-18}$. herefore, we evaluated the cytosol $\mathrm{Ca}^{2+}$ concentration, ROS levels and apoptosis to illustrate the protective effects of Iso on ERS injury. We have found that Isoeffectivelyinhibits ERS-induced GRP78 up-regulation, cytosol $\mathrm{Ca}^{2+}$ overload, ROS burstand apoptosis.

Previous studies have shown that protein kinase $\mathrm{C}$ epsilon (PKCE)can inhibit apoptosis by regulating ROS production and $\mathrm{Ca}^{2+}$ homeostasis. Quercetin also inhibits ROS generation and apoptosis by activating the PKCE signal pathway. Therefore, we hypothesized that Iso, as a metabolite of quercetin, can attenuate ERS-induced ROS burst and $\mathrm{Ca}^{2+}$ overload through the $\mathrm{PKC} \varepsilon$ signal pathway. To test our hypothesis, we applied a PKC $\varepsilon$ specific inhibitor, $\varepsilon V 1-2$, to to approve our hypothesize. As expected, we found that $\varepsilon V 1-2$ reversed the protective effect of Iso on ERS injury in N2a cells. This study was the first to demonstrate that Iso can elicit protective effects against ERS injury in N2a cells and that these effects are mediated, at least in part, via the $\mathrm{PKC} \varepsilon$ pathway.

Iso, one of the flavonoids, has gained considerable attention due to its wide range of biological and pharmacological properties. It has been reported that Iso is efficacious in protecting hepatocytes against oxidative stress by activating Nrf2 and inhibiting ROS production by up-regulating antioxidant protein $\mathrm{HO}-1$ and GCL expression $^{19}$. Iso has anti-inflammatory effects through HO-1activity, which was mediated by the Nrf2/PPAR- $\gamma$ pathway ${ }^{19-21}$. Iso can also activate the NF- $\kappa \mathrm{B}$ signaling pathway to inhibit inflammation and cell proliferation. Additionally, Iso has been shown to inhibit various types of cancer. Ramachandran ${ }^{22}$ found that Iso inhibited the migratory/invasive properties of gastric cancer cells through modulation of the PPAR- $\gamma$ activation pathway. Jaramillo ${ }^{23}$ found that Isoinhibtied colon cancer cells growth by interrupting cell cycle progression. Iso is also found to promote lung cancer cell apoptosis through mitochondriadependent caspase activation in vitro and vivo ${ }^{24}$. Moreover,
Iso can mediate Akt and mitogen-activated protein kinase signal pathways to inhibit cell proliferation and induce apoptosis in breast cancer ${ }^{25}$.

In summary, the therapeutic significance and chemopreventive capabilities of Iso in inflammation, AD and cancer warrant further study. These findings also support further investigation of the pharmacological and molecular mechanisms of other flavonoids on ERS-induced neuron apoptosis.

\section{Acknowledgments}

This work was supported by the National Natural Science Foundation of China (grants 81561019), the Natural Science Foundation Project of Jiangxi Province (grants 20161BAB205214), the Science and Technology Foundation of the Education Department of Jiangxi Province (grants GJJ150759), and a Jinggangshan University grant (grants JZB15006).

\section{References}

1. Huang $Y$, Mucke L. Alzheimer mechanisms and therapeutic strategies. Cell. 2012; 148(6): p. 1204-22.

2. Selkoe DJ. Alzheimer's disease: genes, proteins, and therapy. Physiol Rev. 2001; 81(2): p. 741-66.

3. Alzheimer's A. Alzheimer's disease facts and figures. Alzheimers Dement. 2016; 12(4): p. 459-509.

4. Alzheimer's A. Alzheimer's disease facts and figures. Alzheimers Dement. 2015; 11(3): p. 332-84.

5. Hort J, O'Brien JT, Gainotti G, et al. EFNS guidelines for the diagnosis and management of Alzheimer's disease. Eur J Neurol. 2010; 17(10): p. $1236-48$.

6. Benjannet $\mathrm{S}$, Cromlish JA, Diallo $\mathrm{K}$, et al. The metabolism of betaamyloid converting enzyme and beta-amyloid precursor protein processing. Biochem Biophys Res Commun. 2004; 325(1): p. 235-42.

7. Szegezdi E, Logue SE, Gorman AM, et al. Mediators of endoplasmic reticulum stress-induced apoptosis. EMBO Rep. 2006; 7(9): p. 880-5.

8. Iurlaro R, Munoz-Pinedo C. Cell death induced by endoplasmic reticulum stress. FEBS J. 2016; 283(14): p. 2640-52.

9. Sano R, Reed JC. ER stress-induced cell death mechanisms. Biochim Biophys Acta. 2013; 1833(12): p. 3460-70.

10. Mishra R, Karande AA. Endoplasmic reticulum stress-mediated activation of p38 MAPK, Caspase- 2 and Caspase-8 leads to abrininduced apoptosis. PLoS One. 2014; 9(3): p. e92586.

11. Koeberle A, Pergola C, Shindou H, et al., Role of p38 mitogen-activated protein kinase in linking stearoyl-CoA desaturase-1 activity with endoplasmic reticulum homeostasis. FASEB J. 2015; 29(6): p. 243949.

12. Kim BJ, Ryu SW, Song BJ. JNK- and p38 kinase-mediated phosphorylation of Bax leads to its activation and mitochondrial translocation and to apoptosis of human hepatoma HepG2 cells. J Biol Chem. 2006; 281(30): p. 21256-65.

13. Sovolyova N, Healy S, Samali A, et al. Stressed to death - mechanisms of ER stress-induced cell death. Biol Chem. 2014; 395(1): p. 1-13.

14. Mercado G, Castillo V, Soto P, et al. ER stress and Parkinson's disease: Pathological inputs that converge into the secretory pathway. Brain Res. 2016. 
15. Kiraz Y, Adan A, Kartal Yandim M, et al. Major apoptotic mechanisms and genes involved in apoptosis. Tumour Biol. 2016.

16. Mattson MP. Apoptosis in neurodegenerative disorders. Nat Rev Mol Cell Biol. 2000; 1(2): p. 120-9.

17. Radi E, Formichi P, Battisti C, et al., Apoptosis and oxidative stress in neurodegenerative diseases. J Alzheimers Dis, 2014. 42 Suppl 3: p. S125-52.

18. Bahar E, Kim H, Yoon H. ER Stress-Mediated Signaling: Action Potential and $\mathrm{Ca}(2+)$ as Key Players. Int J Mol Sci. 2016; 17(9).

19. Yang JH, Shin BY, Han JY, et al. Isorhamnetin protects against oxidative stress by activating Nrf2 and inducing the expression of its target genes. Toxicol Appl Pharmacol. 2014; 274(2): p. 293-301.

20. Christensen KB, Petersen RK, Kristiansen K, et al. Identification of bioactive compounds from flowers of black elder (Sambucus nigra L.) that activate the human peroxisome proliferator-activated receptor (PPAR) gamma. Phytother Res. 2010; 24 Suppl 2: p. S129-32.

21. Wang G, Han D, Zhang Y, et al. A novel hypothesis: up-regulation of
HO-1 by activation of PPARgamma inhibits HMGB1-RAGE signaling pathway and ameliorates the development of ALI/ARDS. J Thorac Dis. 2013; 5(5): p. 706-10.

22. Ramachandran L, Manu KA, Shanmugam MK, et al. Isorhamnetin inhibits proliferation and invasion and induces apoptosis through the modulation of peroxisome proliferator-activated receptor gamma activation pathway in gastric cancer. J Biol Chem. 2012; 287(45): p. 38028-40.

23. Jaramillo S, Lopez S, Varela LM, et al. The flavonol isorhamnetin exhibits cytotoxic effects on human colon cancer cells. J Agric Food Chem. 2010; 58(20): p. 10869-75.

24. Lee HJ, Lee HJ, Lee EO, et al. Mitochondria-cytochrome C-caspase-9 cascade mediates isorhamnetin-induced apoptosis. Cancer Lett. $2008 ; 270(2):$ p. 342-53.

25. Hu S, Huang L, Meng L, et al. Isorhamnetin inhibits cell proliferation and induces apoptosis in breast cancer via Akt and mitogenactivated protein kinase kinase signaling pathways. Mol Med Rep. 2015; 12(5): p. $6745-51$. 